\title{
Prácticas Pedagógicas y Transformaciones Sociales. Interculturalidad y Bilingüismo en la Educación de Sordos
}

\author{
Pedagogical Practices and Social Transformations. \\ Interculturality and Bilingualism in the Deaf Education.
}

\author{
Valeria Herrera Fernández ${ }^{1 *}$ \\ Verónica de la Paz Calderón ${ }^{2}$ \\ ${ }^{1}$ Universidad de Chile, Chile \\ ${ }^{2}$ Instituto de la Sodera, Chile
}

\begin{abstract}
El presente estudio aborda la transformación de las prácticas pedagógicas y la generación de respuestas educativas para acoger la diversidad cultural y lingüística de las personas sordas, cuya primera lengua es la Lengua de Señas Chilena. Se considera que los cambios que requiere el sistema educativo ocurren en las aulas y surgen a partir de la reflexión de los profesores que innovan sus prácticas y generan nuevas estrategias de enseñanza del español como segunda lengua para sordos desde un enfoque intercultural bilingüe. Se observaron las prácticas de enseñanza realizadas por 17 profesoras diferenciales de dos escuelas de sordos de la ciudad de Santiago, a partir de una pauta de observación elaborada para dicho fin. Los resultados muestran que las profesoras innovan sus prácticas e implementan estrategias globales (cognitivas y metacognitivas) y específicas (visuales, propias de sordos), con gran potencial transformador. Sin embargo, dichas prácticas y estrategias requieren mayor desarrollo y sistematización.
\end{abstract}

Descriptores: Segunda lengua; Innovación pedagógica; Educación de sordos.

This study deals with the transformation of pedagogical practices and the generation of educational responses to embrace the cultural and linguistic diversity of deaf people whose first language is the Chilean Sign Language. It is considered that the changes required by the education system occur in the classroom and arise from the reflection of teachers who innovate their practices and generate new strategies of teaching Spanish as a second language for the deaf, from a bilingual intercultural approach. Teaching practices of 17 teachers from different special schools for the deaf in the city of Santiago were observed, based on an observation checklist developed for this purpose. The results show that the teachers use cognitive, metacognitive and visual strategies (indigenous of the deaf). However, there is no systematic development of such practices.

Keywords: Second language; Pedagogical practices; Deaf education.

\section{Introducción}

El estudio indaga las prácticas y estrategias de enseñanza usadas habitualmente por profesoras diferenciales que enseñan español a estudiantes sordos, con la finalidad de describirlas, analizarlas y sistematizarlas. A partir de ello, se espera orientar innovaciones didácticas respetando la diversidad lingüística y cultural de los estudiantes, como punto

*Contacto: valherrera@u.uchile.cl

Recibido: 29/06/2018

$1^{\text {a }}$ Evaluación: 25/09/2018

ISSN: 0718-7378

Aceptado: 07/03/2019

www.rinace.net/rlei/ 
de partida para transformar la educación y promover cambios sociales que garanticen los derechos humanos y la participación social de todos.

La superación del analfabetismo en la población sorda y la búsqueda de nuevos modelos de enseñanza ha preocupado durante generaciones a profesores e investigadores de todo el mundo (Herrera, 2009). En los últimos años, se han realizado estudios que proponen prácticas específicas de enseñanza del lenguaje escrito como segunda lengua (Easterbrook, y Beal-Alvarez, 2013; Indesor, 2015; Insor, 2009), basadas en los conocimientos y las epistemologías de sordos (Bauman y Murray, 2014; Moores, 2010; Paul y Moores, 2012) y la Educación Intercultural Bilingüe (EIB). En Chile, la lengua natural de los sordos (L1), es la Lengua de Señas Chilena (LSCH) y la segunda lengua (L2) es el español.

Desde el Congreso de Hamburgo realizado en 1980 se reconoce el derecho de las personas sordas a recibir Educación Bilingüe en su lengua natural. La Organización de Naciones Unidas reconoce este derecho en la Convención de Derechos de las Personas con Discapacidad en 2006, que Chile ratifica en 2008 y reafirma en la Ley 20.422 de Igualdad de Oportunidades e Inclusión Social de las Personas con Discapacidad, promulgada en 2010. Sin embargo, para hacer efectivos estos derechos es necesario transformar las prácticas en las aulas, desarrollar modelos didácticos y sistematizar las buenas prácticas de enseñanza.

El estudio se enmarca en la enseñanza del español como L2 desde la EIB, incorporando la cultura, la lengua y la experiencia visual y gestual propia de los sordos (Benvenuto, 2016; Humphries, 2004; Padden, 2006). Para de la Paz (2012), la interculturalidad es la interacción horizontal entre culturas, a partir de un proceso de comunicación entre grupos humanos diferentes, donde ninguno está por encima del otro, promoviendo la igualdad, la integración y la convivencia armónica entre culturas. Dicha interacción horizontal se sustenta en el respeto a la diversidad y el crecimiento de ambas culturas. La UNICEF (2005) define la educación intercultural como la posibilidad de promover relaciones positivas entre distintos grupos culturales, de confrontar la discriminación, el racismo y la exclusión, de formar ciudadanos conscientes de las diferencias y capaces de trabajar conjuntamente en el desarrollo del país y en la construcción de una democracia justa, igualitaria y plural. En este sentido, la EIB conlleva la integración permanente, transversal y horizontal de dos culturas y dos lenguas en una institución educativa. Poner en práctica la interculturalidad significa pasar de una convivencia entre dos culturas acostumbradas a asumir roles diferentes, a convivir en un espacio donde todos son valorados como seres humanos, con derechos y deberes propios de una comunidad (de la Paz, 2012).

Por otra parte, creemos que los escasos avances en el aprendizaje del lenguaje escrito por parte de los sordos (Moores y Miller, 2009) pueden atribuirse a los métodos de enseñanza utilizados (Herrera, Puente y Alvarado, 2014), ya que en general los profesores utilizan métodos pensados para personas oyentes monolingües, desconociendo la LSCH, la EIB y la necesidad de proveer respuestas educativas que respondan al bilingüismo particular de los sordos. Asimismo, el valor que asignan los profesores a la lengua y la cultura incide en los procesos de enseñanza y en las expectativas académicas acerca de los estudiantes (Cummins, 2002).

Junto con ello, los modelos mentales sobre la lectura tanto de los profesores como de los estudiantes se relacionan con los resultados y se cree que mientras más complejos y flexibles sean estos modelos, mejor será el desempeño lector. Igualmente, las concepciones teóricas y la valoración de la enseñanza determinan las prácticas en las aulas (Crespo, 
García y Carvajal, 2003). Ya que en la medida en que el profesor esté formado teórica y estratégicamente para conducir la enseñanza y aprendizaje, los estudiantes tendrán una visión positiva de la lectura y se acercarán a ella con herramientas apropiadas (Caldera, Escalante y Terán, 2010).

Para Shulman (1987), los saberes pedagógicos de los profesores orientan el proceso de enseñanza y aprendizaje. El quehacer pedagógico entrega información a los docentes para complementar sus prácticas, proporcionándoles información anticipada para articular una clase, programar el tipo de estrategias para facilitar los aprendizajes y desarrollar el pensamiento y la creatividad (Chadwick, 1988). Por todo ello, describir, analizar y sistematizar las prácticas y estrategias de enseñanza usadas por los profesores de sordos en la enseñanza del español como L2, permite explorar los conocimientos pedagógicos con los que cuentan los profesores, orientar la enseñanza y promover cambios en el sistema educativo respecto de la diversidad.

\section{Revisión de la literatura}

En la Educación de Sordos en Chile y en el mundo, predomina una visión patológica de la sordera y enfoques educativos centrados en la oralidad. Desde esta mirada, se concibe la sordera como enfermedad y a los sordos como discapacitados auditivos. Los enfoques educativos derivados de esta perspectiva buscan oralizar a los sordos, sustituyendo las prácticas pedagógicas por prácticas terapéuticas enfocadas en la rehabilitación del habla. En la actualidad, se cuestiona fuertemente esta perspectiva y se promueve, especialmente por los grupos excluidos, una mirada antropológica de las diferencias, entendiendo y valorando la sordera como diversidad biocultural (Bauman y Murray, 2014; Robinson y Henner, 2017).

Comprender la realidad de los sordos desde una mirada antropológica, implica entender las diferencias como diversidad y proveer espacios educativos que reconozcan las múltiples dimensiones de la existencia humana, las similitudes y diferencias culturales y la interculturalidad. Esta mirada realza las experiencias de vida de los sordos desde una realidad visual y gestual, que los lleva a construir conocimientos diferentes de los que construyen los no sordos (Hauser et al., 2010; Holcomb, 2010; Marschark, Tang y Knoors, 2014; Robinson y Henner, 2017).

Desde una perspectiva crítica del conocimiento, en el último tiempo se cuestionan fuertemente las concepciones tradicionales sobre la construcción de conocimientos culturales, proponiendo nuevas epistemologías que reconozcan los conocimientos tradicionales de los grupos minoritarios asimilados por los conocimientos científicos de carácter universal (Olivé, 2009). En este sentido, las Epistemologías de Sordos cuestionan la aplicabilidad de las teorías generales de enseñanza en la educación de sordos (Moores, 2010; Paul y Moore, 2012) y los principios universales del desarrollo y el aprendizaje, emanados de estudios con personas oyentes, aplicados a la población sorda (Humphries, 2004). Para Young y Temple (2014) las personas sordas poseen conocimientos sobre su aprendizaje que deben incorporarse en su educación (Herrera et al., 2014; Muñoz, 2017).

Para Robinson y Henner (2017), la construcción social que define a las personas sordas como discapacitados auditivos, los invalida para contribuir al corpus de conocimientos, debido a la creencia general de que son inherentemente menos inteligentes o capaces. En este sentido, señalan que comprender de qué modo la discapacidad y la lengua sitúan los 
saberes y a quienes conocen, permite evaluar y reformar las estructuras de autoridad epistemológica. Para los sordos, el acceso a la lengua se traduce en acceso al poder y estatus social.

Desde las Epistemologías de Sordos se reconoce la autoridad académica de los sordos, se valora la experiencia de la sordera en la generación de conocimiento educativo y se explicitan las diferencias en las formas de conocer y aprender de los sordos (Paul y Moores, 2012). La enseñanza del español desde este enfoque busca proveer respuestas sociales a las necesidades de aprendizaje de los sordos como estudiantes bilingües, a través de propuestas educativas que consideren las características individuales y las prácticas sociales de la población sorda (Herrera y de la Paz, 2017; Humphries, 2004; Muñoz, 2017).

Innovar las prácticas de enseñanza requiere que los profesores adecuen la situación y los contenidos de aprendizaje, a las necesidades de los estudiantes a partir de diferentes estrategias. Para Easterbrook y Beal-Alvarez (2013), las buenas prácticas de enseñanza de la lectura con sordos requieren al menos cinco componentes; (1) Desarrollar habilidades de pensamiento de orden superior, que implica procesar la información que recibimos y resolver problemas concretos. (2) Comunicación fluida entre profesores y estudiantes para fomentar mejores aprendizajes, ello implica disponer de variadas habilidades comunicativas. (3) Visualizar la información, la organización y los apoyos para mejorar la comprensión, la retención y la memorización. (4) Enseñanza explícita, presentar los objetivos, mostrar la tarea y ejemplificarla, guiar su puesta en práctica y proveer oportunidades para ejercitarla hasta lograrla. (5) Andamiaje y mediación durante la interacción guiada para el logro de los aprendizajes (Vigotsky, 1978).

Un aspecto fundamental de las buenas prácticas se relaciona con el aprendizaje colaborativo, ya que permite aprender con otros y favorece la construcción de conocimientos y el progreso académico. Por su parte, la mediación es necesaria para que los estudiantes puedan integrar los conocimientos nuevos y conectarlos con los aprendidos para luego aplicarlos. Los aprendizajes significativos requieren que los profesores sean mediadores de los conocimientos, orienten el logro de los objetivos, provean los apoyos necesarios, planteen desafíos cognitivos alcanzables para mantener la motivación y faciliten oportunidades de éxito en la tarea.

En el mismo sentido, Lissi, Svartholm y González (2012) señalan que una buena práctica pedagógica en un contexto EIB, requiere considerar las estrategias de enseñanza a utilizar, el entorno donde se realiza la clase, la ubicación espacial de los estudiantes (de manera que todos vean y se vean entre sí), mantener los materiales a disposición de los estudiantes y estructurar, organizar y contextualizar la clase de manera visual. Señalan que al comenzar la clase es importante entablar un diálogo con los estudiantes sobre los temas vistos previamente o recordar alguna actividad significativa relacionada con la clase que realizarán, formular preguntas e introducir el nuevo tema.

En relación con las estrategias de lectura y escritura, Gaskins y Elliot (1999) las definen como estrategias cognitivas que apoyan los procesos de pensamiento y exigen un compromiso activo por parte del estudiante para construir sentido, adquirir dominio de la comprensión y recordar conceptos. Por su parte, Dole, Nokes y Drits (2009) especifican que las estrategias cognitivas son aquellas referidas a procesos dinámicos y constructivos que el lector debe realizar de forma consciente e intencional para construir una representación mental del texto escrito. 
Gaskins y Elliot (1999) proponen dos tipos principales de estrategias; las estrategias para alcanzar el sentido y recordarlo y las estrategias para producir conocimientos. En este estudio, hemos denominado estrategias globales de lectura a las estrategias que permiten alcanzar sentidos y recordarlos, como: (1) Acceder a las experiencias previas para contextualizar la lectura, aprovechar los conocimientos del mundo, de la LSCH y el vocabulario con que cuentan sobre el tema. (2) Anticipar, formular hipótesis, preguntas y expectativas sobre el texto antes de leer para contextualizar y favorecer la comprensión. (3) Explicitar los propósitos de la lectura y utilizar un guion de la clase para que los estudiantes puedan seguirla o retomarla, formular objetivos posibles de lograr en una clase y establecer continuidad con nuevos objetivos. (4) Interesar y motivar a los estudiantes a la lectura, explorar las imágenes, el texto y el título y anticipar el tema. (5) Generar esquemas y organizadores visuales para presentar y visualizar la información, seguir la clase, anticipar situaciones e identificar ideas principales. (6) Presentar el texto a toda la clase para visualizar la información y guiar la lectura colectiva. (7) Realizar lectura silenciosa global antes de leer para familiarizar a los estudiantes con el tema y el vocabulario. (8) Realizar anticipaciones, hipótesis y formular expectativas sobre el texto durante la lectura para mantener la atención y verificar la comprensión. (9) Realizar preguntas globales en LSCH para comprobar la comprensión, responder preguntas y alcanzar consensos, promoviendo la reflexión metalingüística a partir de la LSCH. (10) Secuenciar los eventos más importantes y/o detener la interrogación del texto para comprobar la comprensión y retomar las ideas principales. (11) Mantener la motivación a través de comentarios sobre la lectura relacionando el tema con sus experiencias de vida y la realidad. (12) Utilizar textos auténticos como libros, periódicos, revistas, cartas, afiches, etc. que permitan interrogar textos complejos relacionados con los intereses de los estudiantes y los contenidos del currículo. (13) Elaborar resúmenes en LSCH y escribirlos, mostrando a los estudiantes las diferencias lingüísticas entre L1 y L2. (14) Promover la lectura colectiva, donde cada estudiante sienta que aporta a la comprensión, aprende de los errores con naturalidad y aprende de y con sus pares.

Junto con ello, para alcanzar el sentido del texto y recordarlo, proponemos estrategias específicas de lectura, por ser estrategias que consideran la singularidad de los estudiantes sordos como aprendices visuales de una L2. Entre ellas: (1) Realizar preguntas de inferencia en $\mathrm{LSCH}$ respecto de la información implícita y explícita, descubriendo lo implícito en el texto y cómo extraer esta información. (2) Explicitar las diferencias entre L1 y L2, puesto que las gramáticas de ambas lenguas son diferentes. (3) Vincular imagen, seña y palabra para favorecer la memorización de palabras y la comprensión. (4) Asociar seña, palabra y dactilología (secuencias de encadenamiento) para asociar la información visual de L1 y L2. (5) Realizar marcas visuales a los textos para apoyar los significados, destacar información importante y entregar pistas sobre las estructuras gramaticales. (6) Utilizar la dactilología para construir y ampliar significados. (7) Marcar la ortografía y la morfología para construir significados y entregar pistas sobre las relaciones morfológicas de las palabras (prefijos, sufijos, etc.). (8) Usar la desinencia verbal para profundizar significados y sistematizar aspectos de la gramática y la sintaxis de la L2. (9) Conectar información entre oraciones y párrafos manteniendo continuidad en la lectura. (10) Trabajar vocabulario visual, signado y escrito para ampliar el léxico y las oportunidades multimodales de acceso al almacén léxico. (11) Ordenar sintácticamente frases u oraciones, realizando ejercicios de estructuración sintáctica para identificar las partes de la oración y construir y reconstruir significados. (12) Usar diccionarios de significados, de sinónimos 
y antónimos, etc. para buscar información y motivar la participación en la construcción de conocimientos. (13) Buscar información en las aulas, en la web, en libros, en cuadernos y entre sus pares para mantener la motivación y desarrollar estrategias de resolución de problemas. (14) Realizar lectura compartida para motivar, conversar, opinar sobre lo leído y dar y recibir recomendaciones sobre un texto.

En cuanto a las estrategias para producir conocimiento planteadas por Gaskins y Elliot (1999), en el estudio las denominamos estrategias globales para la escritura. Como: (1) Escribir para una gran variedad de propósitos, estableciendo previamente para qué y por qué se escribe. (2) Escribir diferentes tipos de textos y propósitos para conocer las características de los textos según su finalidad. (3) Narrar en LSCH las ideas que se quieren comunicar y luego escribirlas en español. (4) Explicitar las diferencias entre LSCH y español durante la escritura para mostrar la estructura de cada lengua. (5) Revisar y corregir los textos escritos utilizando LSCH para monitorear la formulación de las ideas y la compresión de lo escrito en su L1. (6) Numerar temas posibles de escribir a partir de las experiencias y conocimientos previos, escribir lluvia de ideas, listar temas y subtemas y organizar la información. (7) Realizar primeros borradores, revisiones y publicación, tanto de forma individual, en parejas o en grupos. (8) Reflexionar sobre los textos escritos, conversar sobre la escritura y las estrategias utilizadas, motivando la escritura de futuros textos.

Igualmente, hemos planteado estrategias específicas para producir conocimiento a las que hemos denominado estrategias específicas de escritura, por ser estrategias propias de los sordos como aprendices visuales. Entre ellas: (1) Relatar las ideas en LSCH para planificar la escritura, conversar en LSCH las ideas y características del texto y luego escribirlas. (2) Usar marcadores visuales como ayuda para estructurar frases y oraciones. (3) Presentar material visual para apoyar la escritura y conversar sobre las imágenes. (4) Usar la dactilología para aumentar el vocabulario y la memorización de palabra desde múltiples modalidades. (5) Analizar errores en la escritura de forma colectiva para favorecer el aprendizaje a partir del error y tomar conciencia de las diferencias entre las lenguas. (6) Realizar actividades grupales de revisión y corrección de la escritura, valorar la reflexión colectiva para enriquecer el conocimiento de la L2. (7) Realizar actividades de escritura compartida de manera que cada estudiante tenga la oportunidad de aportar, enriquecer la escritura y aprender de y con sus compañeros. (8) Compartir y socializar la escritura para mejorar la autoestima y el gusto por la escritura.

\section{Método}

El estudio forma parte de una investigación mayor ${ }^{1}$ de carácter cuantitativo que pretende sacar a la luz las relaciones causa-efecto entre un fenómeno y sus determinantes (Giroux y Tremblay, 2004; McMillan y Schumacher, 2005). El diseño se justifica por ser el más indicado cuando la investigación cuantitativa se desarrolla en escenarios educativos naturales, no es posible hacer asignación al azar de los sujetos, ni control completo de las variables. El presente estudio provee los insumos (estrategias pedagógicas) para sistematizar las características de una metodología de enseñanza de la lectura para sordos,

${ }^{1}$ Fondecyt Regular $\mathrm{N}^{\circ} 1130182$ "Comprensión lectora en estudiantes sordos de $1^{\circ}$ a $4^{\circ}$ año básico. Estrategias visuales, cognitivas y metacognitivas y prácticas efectivas de enseñanza” (2013-2016). 
a partir de estrategias de aprendizaje visual y prácticas educativas centradas en la visualidad de los estudiantes (cuadro 1).

Cuadro 1. Pauta de observación sobre prácticas y estrategias de enseñanza

SI $\quad$ NO

I. Estrategias Globales de Lectura

1. Incorpora la experiencia previa y activa conocimientos vinculados al texto.

2. Genera anticipaciones, hipótesis y expectativas sobre el texto antes de leer.

3. Explicita los propósitos de la lectura.

4. Actividades de motivación a la lectura.

5. Esquema y organizadores visuales de la clase que los niños pueden seguir.

6. Texto a toda la clase (pizarra, proyector, etc.)

7. Lectura silenciosa global.

8. Anticipaciones, hipótesis y expectativas sobre el texto durante la lectura.

9. Preguntas globales en LSCH para comprobar la comprensión.

10. Secuencia de los eventos más importantes para monitorear la comprensión.

11. Mantiene la motivación a través de comentarios sobre la lectura.

12. Textos auténticos (libros, diarios, cartas, afiches, etc.)

13. Resúmenes de lo leído en LSCH y/o por escrito.

14. Lectura colectiva

II. Estrategias Específicas de Lectura

1. Preguntas de inferencia respecto de información implícita y explícita.

2. Realiza diferencias entre LSCH y español cuando lo amerita.

3. Asocia imagen, signo y palabra.

4. Asocia signo, palabra y dactilología (secuencias chaining).

5. Marcadores visuales para apoyar significados.

6. Alfabeto manual /dactilología para construir y ampliar significados.

7. Ortografía y morfología para construir significados.

8. Desinencia verbal (persona, tiempo) para profundizar significados.

9. Conexiones entre oraciones y párrafos.

10. Trabaja vocabulario (visual, signado, escrito) para ampliar el léxico.

11. Ordenar sintácticamente frases u oraciones.

12. Usa diccionarios de sinónimos y antónimos.

13. Búsqueda de información.

14. Lectura compartida.

III. Estrategias Globales de Escritura

1. Escritura para una gran variedad de propósitos.

2. Escritura de diferentes tipos de textos y propósitos.

3. Escritura a partir de LSCH.

4. Diferencias de estructura en LSCH y español en la escritura.

5. Revisión y corrección de los textos escritos utilizando LSCH.

6. Numerar temas posibles de escribir.

7. Realización de primeros borradores, revisiones, edición y publicación.

8. Actividades de reflexión de los textos escritos.

IV. Estrategias Específicas de Escritura

1. Narración en LSCH para planificar la escritura.

2. Marcadores visuales para estructurar frases y oraciones.

3. Material visual como apoyo.

4. Dactilología para memorizar la escritura de palabras.

5. Analizar errores en la escritura de forma colectiva.

6. Actividades grupales de revisión y corrección de la escritura.

7. Actividades de escritura compartida.

8. Compartir y socializar las producciones escritas.

Fuente: Elaboración propia.

Se formula como una investigación útil para la educación relacionando teoría y práctica. Esta perspectiva considera que los problemas en educación se resuelven actuando educativamente en situaciones variadas y complejas (Gordillo, 1992). La investigación se 
aborda desde un diseño descriptivo (Pérez Rodríguez et al., 1996), que permite observar las prácticas y estrategias usadas por los profesores en la enseñanza del español como L2. Asumimos que los resultados de una investigación de estas características no constituyen una respuesta concluyente ni refutan ninguna hipótesis, pero constituyen una herramienta fundamental para analizar el problema de investigación.

El estudio busca comprender ¿ccómo enseñan a leer y a escribir los profesores de sordos a sus estudiantes y cuáles son sus prácticas pedagógicas y estrategias de enseñanza?, con el objetivo de describirlas y analizarlas desde una perspectiva de enseñanza de L2. Las participantes del estudio fueron 17 profesoras entre 24 y 60 años, especialistas en audición y lenguaje, con diferentes recorridos profesionales, formación inicial docente en distintas instituciones y diferentes niveles de LSCH. Esta diversidad profesional la encontramos habitualmente en las aulas de sordos en Chile. Las profesoras firmaron voluntariamente consentimientos informados aceptando ser observadas en sus clases de lengua. En total, se realizaron 25 horas y 30 minutos de observación directa en las aulas.

Se utilizó la observación ya que permite establecer una relación concreta e intensiva entre las investigadoras y las profesoras como actores sociales (McMillan y Schumacher, 2005). Se observaron las prácticas pedagógicas en el medio natural donde ocurren para luego analizarlas en relación con los conocimientos establecidos (Bisquerra, 2004). La observación fue estructurada en una pauta de cotejo compuesta por 4 categorías y 44 subcategorías, establecidas con anterioridad en base a evidencia teórica y práctica (cuadro 1). Dicha pauta fue validada por el juicio de seis expertos en enseñanza de la lectura. Se estableció una escala de calificación para consignar la presencia o ausencia de la estrategia. Las categorías establecidas son (1) estrategias globales de lectura, (2) estrategias específicas de lectura, (3) estrategias globales de escritura y (4) estrategias específicas de escritura.

\section{Resultados}

Las estrategias globales de lectura corresponden a estrategias cognitivas y metacognitivas que permiten generar un contexto de aprendizaje significativo a partir de las experiencias previas y el conocimiento de mundo. En este sentido, las principales estrategias observadas fueron realizar preguntas generales en LSCH, para verificar la comprensión y presentar el texto a toda la clase, ambas usadas por 16 profesoras $(94,1 \%)$. En segundo lugar, trabajar con textos auténticos, usada por 12 profesoras (70,0\%). Motivar el interés por la lectura y mantenerlo durante la clase, fue una estrategia observada en 11 profesoras $(64,7 \%)$. Incorporar la experiencia previa y activar los conocimientos en la interrogación de texto, se observó en las prácticas de 10 profesoras (58,8\%). La elaboración de resúmenes de lo leído en LSCH o de forma escrita para ampliar la comprensión, se observó en 10 profesoras $(58,8 \%)$ y el uso de esquemas y organizadores visuales se observó en 5 profesoras $(29,4 \%)$.

Respecto a las estrategias específicas de lectura, estas corresponden a las estrategias de sordos que Padden (2006) denomina indigenous strategies. Estas son estrategias que requieren mayor desarrollo e implementación pedagógica y que en esta investigación se exploran y sistematizan (figura 1). La implementación didáctica de estas estrategias promueve la lectura a través del desarrollo de las potencialidades y características de 
aprendizaje visual de los sordos. Esta perspectiva permite aprovechar el conocimiento en L1 para producir textos en L2 (Cummins, 2002).

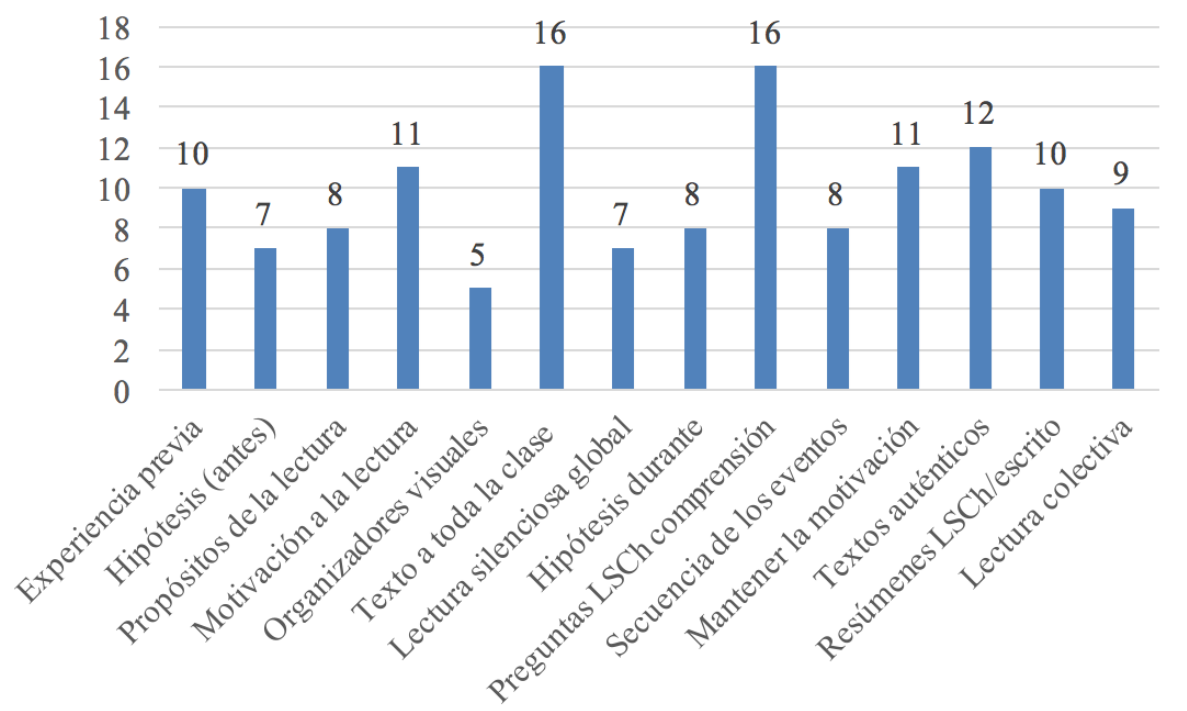

Figura 1. Número de profesoras y estrategias globales de lectura Fuente: Elaboración propia.

La principal estrategia específica de lectura observada en las prácticas de las profesoras fue el trabajo de vocabulario visual, signado y/o escrito en un 94,1 \% (16 profesoras). En segundo lugar, el uso del alfabeto dactilológico para construir y ampliar significados en un $82,3 \%$ (14 profesoras). En tercer lugar, y con igual porcentaje (82,3\%), la asociación de imágenes, señas y palabras; señas, palabras y dactilologías y usar la ortografía y la morfología para construir significados, en un $64,7 \%$ (11 profesoras).

La realización de preguntas de inferencia sobre la información implícita y explícita, el trabajo con las desinencias gramaticales para profundizar significados y la ordenación sintáctica de frases y oraciones, fueron observadas en 10 profesoras $(58,8 \%)$. La lectura compartida se observó en las clases de 9 profesoras $(52,9 \%)$, el uso de diccionarios en 8 profesoras $(47,1 \%)$, la búsqueda de información y la explicitación de las diferencias entre LSCH y español en 6 profesoras $(35,2 \%)$. Las estrategias menos observadas fueron conectar frases y oraciones y establecer un hilo conductor durante la lectura (3 profesoras).

Las estrategias globales de escritura son estrategias cognitivas y metacognitivas, que permiten producir textos a partir del conocimiento de la LSCH (que permite pensar la escritura en L1) para posteriormente escribir las ideas en L2. Esta perspectiva aprovecha el conocimiento en LSCH para producir textos en español, entendiendo que la experiencia cognitiva es abstracta, subyace a las lenguas y se interrelaciona (Cummins, 2002). En este sentido, las principales estrategias observadas fueron escribir para una gran variedad de propósitos, pensar las ideas en LSCH antes de escribir y revisar y corregir la escritura usando LSCH, usada por 7 profesoras $(41,1 \%)$. La enseñanza explicita de las diferencias lingüísticas entre L1 y L2 se observó en 5 profesoras (29.4\%) y escribir diferentes tipos de textos, numerar temas para escribir y realizar borradores, revisiones y publicación de los textos producidos por los estudiantes se observó en 3 profesoras (figura 2). 


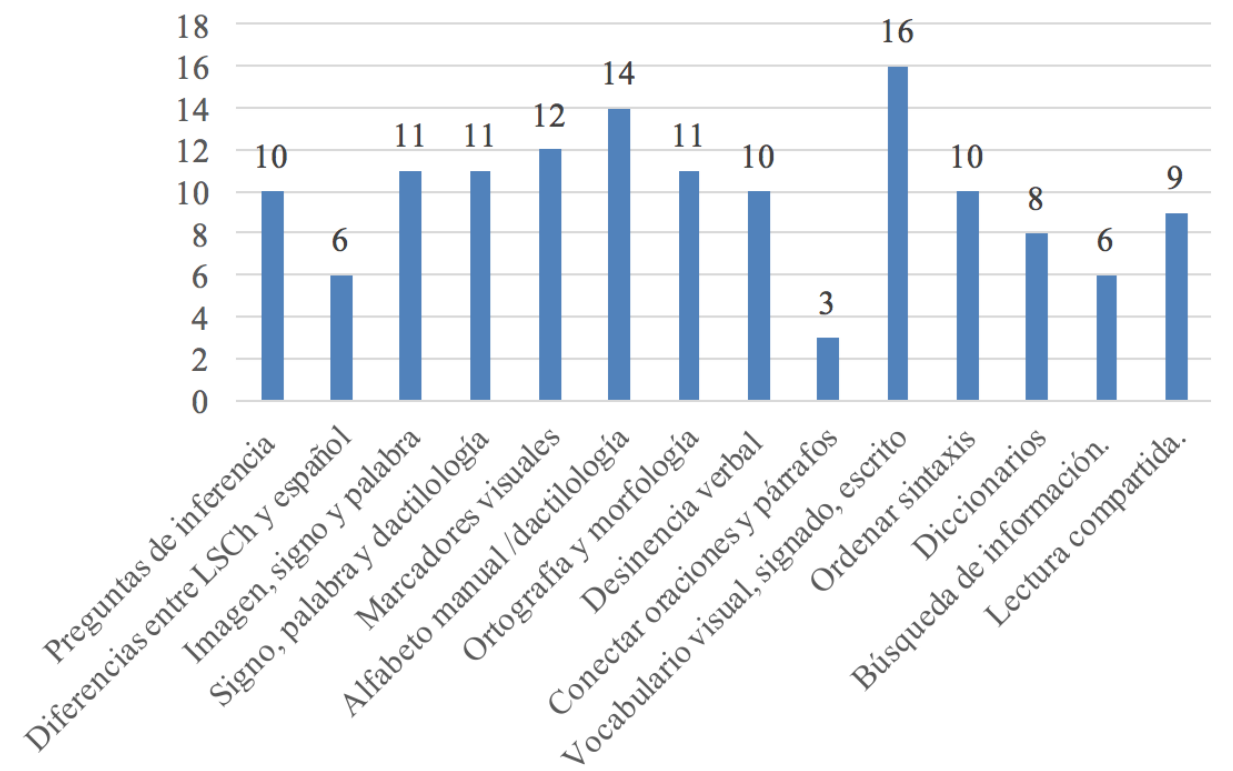

Figura 2. Número de profesoras y estrategias específicas de lectura Fuente: Elaboración propia.

Las estrategias específicas de escritura son estrategias propias (Padden, 2006) de sordos y se utilizan para favorecer la escritura (figura 3). En primer lugar, las estrategias más usadas son el uso de material visual como apoyo y la dactilología para memorizar la escritura de palabras en un 64,7\% (11 profesoras). En segundo lugar, la narración en LSCH para planificar la escritura y analizar errores en la escritura de forma colectiva en un $41,1 \%$ (7 profesoras). En tercer lugar, el uso de marcadores visuales para estructurar frases y oraciones y actividades grupales de revisión y corrección de la escritura en un 35,2\% (6 profesoras). Las estrategias específicas de escritura que observamos con menor frecuencia son la escritura compartida (4 profesoras) y la socialización de las producciones escritas (3 profesoras).

La sistematización de las prácticas de enseñanza observadas durante más de 25 horas muestra que las profesoras focalizan su quehacer en la lectura, observando escasas instancias para la escritura. En general, se observa con frecuencia el uso de estrategias cognitivas y metacognitivas (globales), tanto en la enseñanza de la lectura como en la escritura. Sin embargo, son escasas las instancias en que se observa la implementación de estrategias específicas más coherentes con la visualidad de los sordos. Esta observación, refuerza la necesidad de contar con conocimientos pedagógicos y didácticos específicos para la enseñanza del español como L2 a estudiantes sordos (figura 4). 


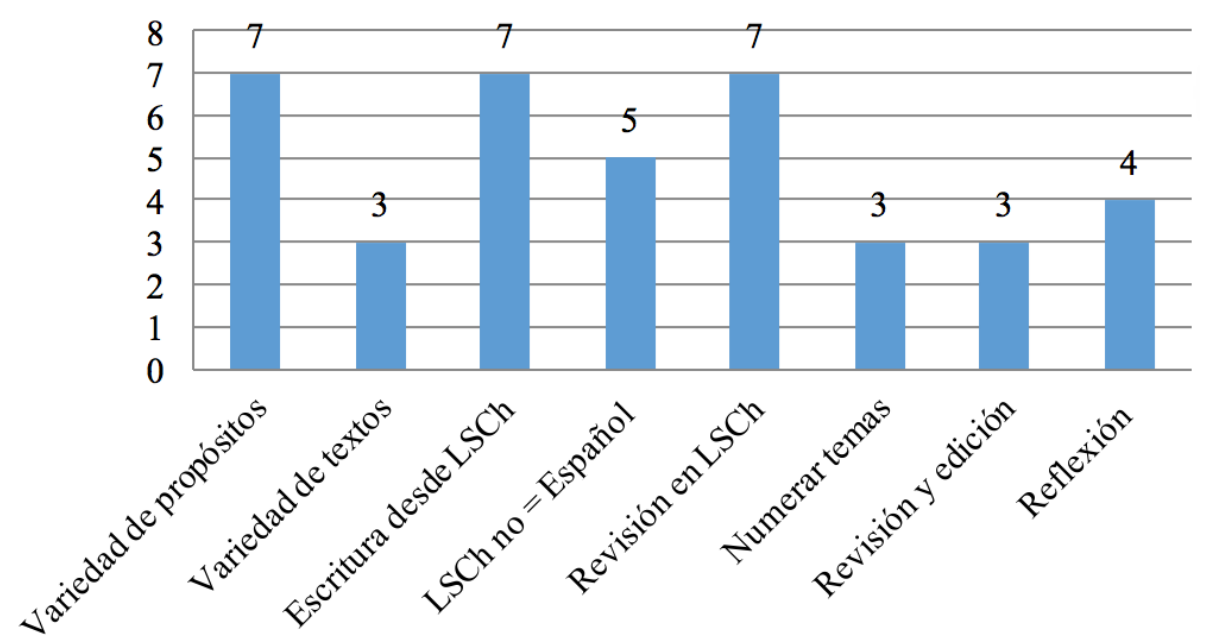

Figura 3. Número de profesoras y estrategias globales de escritura Fuente: Elaboración propia.

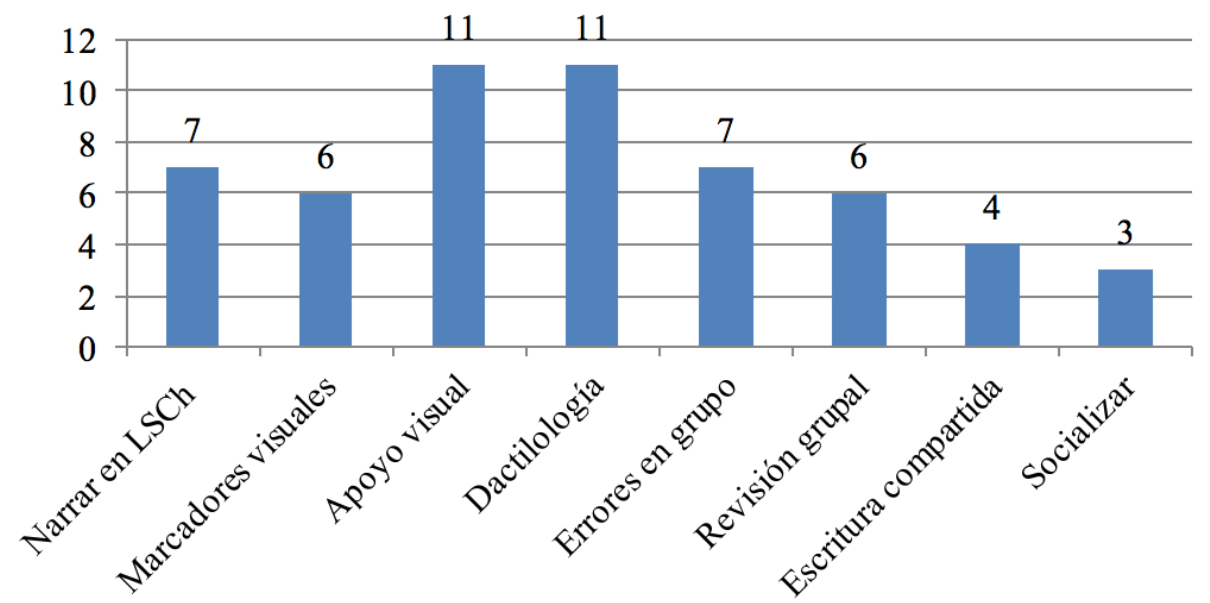

Figura 4. Número de profesoras y estrategias específicas de escritura Fuente: Elaboración propia.

\section{Conclusiones}

El estudio permite aproximar las concepciones teóricas sobre la discapacidad, la sordera y la educación, a las prácticas de enseñanza del español como L2 a estudiantes sordos. Los cambios paradigmáticos transforman el quehacer docente y requieren innovaciones didácticas para responder a las necesidades de aprendizaje de los estudiantes y dar derecho, en toda regla, a la educación. Abordar esta problemática social, implica analizar diversos elementos, uno de ellos se refiere a incorporar en las prácticas pedagógicas la visualidad de los sordos, pues a través de ella se relacionan con el mundo y construyen conocimientos (Chamberlain, Morford y Mayberry, 2000; Hauser et al., 2010; Herrera, 2014; Herrera y de la Paz, 2017; Marschark y Hauser, 2012; Moores, 2010). La visualidad y gestualidad de la LSCH son elementos constitutivos de la identidad y el bilingüismo de los sordos, fundamentales en su desarrollo emocional, social y académico. En este sentido, comprender la sordera como una enfermedad o una limitación a subsanar conduce a 
prácticas colonizadoras fonocéntricas (Robinson y Henner, 2017), que conciben la enseñanza del español a los sordos como la enseñanza de una primera lengua, la lengua oral, dificultando aún más su aprendizaje.

En general, las prácticas pedagógicas y las estrategias de enseñanza usadas por los profesores de sordos, parecen reproducir las teorías, modelos y didácticas usadas con estudiantes oyentes (Herrera et al., 2014; Paul y Moores, 2010; Robinson y Henner, 2017). En el caso de la enseñanza de la lectura y la escritura, esta reproducción niega las características de aprendizaje visual de los sordos y subvalora su lengua y cultura al no incorporarlas en el currículum escolar (Cummins, 2002). Esta situación obstaculiza la generación de conocimientos pedagógicos y didácticas específica para la enseñanza del español como L2 a estudiantes sordos (Marschark et al., 2014; Young y Temple, 2014).

El proceso de enseñanza y aprendizaje del español como L2 para estudiantes sordos, es complejo y altamente especializado. Por ello, resulta fundamental contar con estrategias de enseñanza globales (basadas en la cognición y metacognición) y específicas (propias de sordos), implementadas pedagógicamente desde la visualidad. Junto con ello, es necesario desarrollar conocimientos teóricos y prácticos respecto de su aplicación en cada una de las distintas tareas que demanda el proceso y contar con secuencias didácticas para orientarlo.

La enseñanza del español como L2 requiere que los docentes se cuestionen previamente qué enseñar, cuáles son los aprendizajes previos para el logro del objetivo, y en base a esto, anticipen las estrategias que requieren (Chadwick, 1988), puesto que el conocimiento pedagógico de los profesores permite orientar los procesos de enseñanza a través de un saber pedagógico (Shulman, 1987). En este proceso, L1 y L2 están presentes permanentemente, pero es fundamental abordarlas pedagógicamente de forma separada para no confundir a los estudiantes y utilizar las estrategias específicas para el desarrollo de cada una de las lenguas.

Al igual que Lissi, Svartholm y González (2012) hemos observado la importancia de distinguir ambas lenguas y explicitar sus diferencias. Al respecto, hemos notado que leer un párrafo u oración y luego solicitar al estudiante que relate lo leído en LSCH facilita el aprendizaje, permite verificar la comprensión y es una oportunidad para explicitar las diferencias entre lenguas. Por otra parte, el uso frecuente de información visual como mapas conceptuales, gráficos e imágenes, tanto para introducir un tema como para finalizarlo, permite a los estudiantes contextualizar los aprendizajes y memorizar visualmente lo trabajado en clases, a través de una especie de play mental (Muñoz, 2017). Enfrentar un texto sin estos apoyos dificulta la comprensión y desmotiva el aprendizaje.

Observamos que la escritura se trabaja poco en las aulas. Sin embargo, su desarrollo es fundamental para los estudiantes y precisa que los profesores conozcan las diferentes etapas de una producción escrita (planificación, revisión y edición), las características del tipo de texto a producir y la gramática que van a enseñar. Abordar la enseñanza de la lectura y la escritura a estudiantes sordos desde un EIB requiere que los profesores cuenten con conocimientos lingüísticos tanto de LSCH como de español. Al escribir, resulta fundamental establecer estrategias de revisión y corrección para mejorar la redacción, abordar los errores para aprender de ellos, fortalecer la autoestima y mantener la motivación. En el caso de los sordos, la sistematización de las estrategias de producción escrita, la enseñanza explícita y la evaluación se comprenden de forma distinta cuando se asumen desde una perspectiva de segunda lengua, permite ampliar las oportunidades de 
aprendizaje, promover la reflexión metalingüística en ambas lenguas y mejorar los aprendizajes (Herrera, Chacón y Saavedra, 2016).

Si aplicamos los planteamientos teóricos de Cummins (2002) a la educación de sordos, el bilingüismo implica reconocer la LSCH como L1 y considerarla en los programas de enseñanza del español como L2. En Chile, los propios sordos señalan que la LSCH es fundamental en la enseñanza del español en la educación de sordos (Herrera, Jiménez y Alvarado, 2015; Muñoz, 2017). Implementar programas de EIB para estudiantes sordos conlleva revisar las concepciones de discapacidad, de enseñanza y de enseñanza de segunda lengua, explorando cómo el conocimiento de la LSCH se relaciona con el aprendizaje del español. Por otra parte, y como señalan Piñar, Dussias y Morford (2011), comprender las diferencias entre bilingües sordos y bilingües oyentes, podría enriquecer los actuales modelos de bilingüismo y contribuir al conocimiento sobre cómo el bilingüismo afecta la cognición.

Una de las transformaciones sociales que requiere la comunidad sorda para participar en una sociedad más inclusiva y diversa, es innovar las prácticas pedagógicas desde la visualidad, el bilingüismo y la interculturalidad de los estudiantes. Las prácticas de los profesores que asumen la EIB se derivan de estos principios, se centran en los estudiantes y promueven entornos sociales enriquecidos en las aulas a partir de estrategias multilingüísticas y multiculturales (Easterbrook y Beal-Álvarez, 2013). Como señala Walsh (2005) la interculturalidad es mucho más que el contacto entre culturas, es un intercambio equitativo en condiciones de igualdad. Constituye una meta social por alcanzar y debe ser entendida como un proceso permanente de relación, comunicación y aprendizaje entre personas, grupos, conocimientos, valores y tradiciones, orientada a generar, construir y propiciar el respeto mutuo y el pleno desarrollo de las capacidades de los individuos, por encima de sus diferencias culturales y sociales. La interculturalidad intenta romper con la historia hegemónica de una cultura dominante y otras subordinadas, reforzando las identidades tradicionalmente excluidas para construir una convivencia de respeto y legitimidad entre todos los grupos de la sociedad.

Finalmente, esperamos que esta investigación contribuya al esfuerzo cotidiano que realizan los profesores, proponiendo prácticas y estrategias que respondan a la singularidad de los procesos de aprendizaje del español como L2 de estos estudiantes. Para avanzar en la interculturalidad y el bilingüismo, la educación requiere que los profesores transformen sus prácticas de enseñanza y respondan pedagógica y estratégicamente a los problemas sociales que generan exclusión y discriminación.

\section{Agradecimientos}

Agradecemos a las profesoras que participaron en el estudio y que generosamente compartieron sus experiencias con nosotras. Además, al Fondo Nacional de Ciencia y Tecnología por Proyecto Fondecyt Regular $\mathrm{N}^{\circ} 1130182$ "Comprensión lectora en estudiantes sordos de $1^{\circ}$ a $4^{\circ}$ año básico. Estrategias visuales, cognitivas y metacognitivas y prácticas efectivas de enseñanza” (2013-2016). 


\section{Referencias}

Bauman, H. L. y Murray, J. (2014). Deaf gain: Raising the stakes for human diversity. Minneapolis, MN: University of Minnesota Press.

Bisquerra, R. (2004). Metodología de la investigación educativa. Madrid: La Muralla.

Benvenuto, A. (2016). Entre deficiencias y diferencias. ¿Qué lugar para la igualdad? Reflexiones sobre los sordos para uso de los que hablan y oyen. Santiago de Chile: Universidad de Chile.

Caldera, R. Escalante, D. y Terán, D. (2010). Práctica pedagógica de la lectura y formación docente. Revista de Pedagogía, 31(8), 15-37.

Cummins, J. (2002). Lenguaje, poder y pedagogía. Madrid: Morata.

Crespo, N., García G. y Carvajal, C. (2003). Concepciones didácticas de la lectura y su influencia en el saber de los escolares. Onomazein, 8, 161-174.

Chadwick, C. (1988) Estrategias cognitivas y afectivas de aprendizaje. Revista Latinoamericana de Psicología, 20(2), 163-184.

Chamberlain, C., Morford, J. P. y Mayberry, R. (Eds.) (2000). Acquisition of language by eyes. Londres: Erlbaum.

de la Paz, M. V. (2012). Hacia la búsqueda de la cultura sorda en Chile. Propuesta intercultural bilingüe para sordos. Madrid: Editorial Académica Española.

Dole, J. A., Nokes, J. D. y Drits, D. (2009). Cognitive strategy instruction. En G. G. Duffy y S. E. Israel (Eds.), Handbook of research on reading comprehension (pp. 347-372). Nueva York, NY: Erlbaum.

Easterbrook, S. y Beal-Alvarez, J. (2013). Literacy instruction for students who are deaf and hard of hearing. Nueva York, NY: Oxford University Press.

Gaskins, I. y Elliot, T. (1999). Cómo enseñar estrategias cognitivas en la escuela. Madrid: Paidos.

Giroux, S. y Tremblay, G. (2004). Metodología de las ciencias humanas. La investigación en acción. Ciudad de México: Fondo de Cultura Económica.

Gordillo, M. V. (1992). El enfoque científico y la investigación en educación: La búsqueda de una nueva metodología. Revista Complutense de Educación, 3(2), 180-192.

Hauser P., O’Hearn A., McKee M., Steider, A. y Thew, D. (2010). Deaf epistemology: Deafhood and deafness. American Annals of the Deaf, 154(5), 486-492.

https://doi.org/10.1353/aad.0.0120

Herrera, V. (2009). En busca de un modelo educativo y de lectura coherente con las necesidades educativas especiales de los estudiantes sordos. REXE. Revista de Estudios y Experiencias en Educación, 8(16), 11-24.

Herrera, V. (2014). Alfabetización y bilingüismo en aprendices visuales. Aportes desde las epistemologías de sordos. Revista Educación y Educadores, 17(1), 135-148.

Herrera, V. Chacón, D. y Saavedra, F. (2016). Evaluación de la escritura de estudiantes sordos bilingües. Revista Estudios Pedagógicos, 42(2), 171-191. https://doi.org/10.4067/So718-07052016000200010

Herrera, V., Jiménez, A. y Alvarado, J.M. (mato, 2015). Literacy experiences and learning strategies reader. Tales of deaf adults. Comunicacion presentada en el 22nd International congress on the education of the deaf. Universidad de Patras. 
Herrera V., Puente, A. y Alvarado, J. M. (2014). Visual learning strategies to promote literacy skills in prelingually deaf readers. Revista Mexicana de Psicología, 31(1), 1-10.

Holcomb, T. (2010). Deaf epistemology: The deaf way of knowing. American Annals of the Deaf, 154(5), 471-478. https://doi.org/10.1353/aad.0.0116

Humphries, T. (2004). The modern deaf self: Indigenous practices and educational imperatives. En B. Brueggemann (Ed.), Literacy and deaf people. Cultural and contextual perspectives. Washington, DC: Gallaudet University Press.

Insor. (2009). La enseñanza de la lengua escrita como segunda lengua en la básica primaria. Bogotá: Ministerio de Educación Nacional.

Indesor. (2014). 10 años de bilingüismo en Chile: Experiencias pedagógicas de la escuela intercultural bilingüe para estudiantes sordos. Santiago de Chile: Universidad Metropolitana de Ciencias de la Educación.

Lissi, M. A., Svartholm, K. y González, M. (2012). El enfoque bilingüe en la educación de sordos: Sus implicancias para la enseñanza y aprendizaje de la lengua escrita. Revista Estudios Pedagógicos, 38(2), 299-320. https://doi.org/10.4067/So7 18-07052012000200019

McMillan, J. H. y Schumacher, S. (2005). Investigación educativa. Una introducción conceptual. Madrid: Pearson Addison Wesley.

Marschark, M. y Hauser, P. (2012). How deaf children learn. What parents and teachers need to know. Nueva York, NY: Oxford University Press.

Marschark, M., Tang, G. y Knoors, H. (2014). Bilingualism and bilingual deaf education. Nueva York, NY: Oxford University Press.

https://doi.org/10.1093/acprof:oso/9780199371815.001.0001

Moores D. (2010). Epistemologies, deafness, learning, and teaching. American Annals of the Deaf, 154(5), 447-455. https://doi.org/10.1353/aad.0.0123

Moores, D. y Miller, M. (2009). Deaf people around the world. Educational, development and social perspectives. Washington, DC: Gallaudet University Press.

Muñoz, K. (2017). Concepciones educativas de jóvenes sordos. La experiencia de la sordera como base para la generación de conocimiento pedagógico. Tesis Doctoral. Universidad de la Frontera.

Olivé, L. (2009). Por una auténtica interculturalidad basada en el reconocimiento de la pluralidad epistemológica. En C. Costa y L. López (Eds.), Pluralismo epistemológico (pp. 19-30). Buenos Aires: Clacso.

Organización de Naciones Unidas. (2006). Convención de derechos de personas con discapacidad. Washington, DC: Organización de Naciones Unidas.

Padden, C. (2006). Learning fingerspelling twice: Young signing children's acquisition of fingerspelling. En M. Marschark, B. Schick y P. Spencer (Eds.), Advances in sign language development by deaf children (pp.189-201). Nueva York, NY: Oxford University Press.

Paul, P. V. y Moores, D. (2010). Perspectives on deaf epistemologies. American Annals of the Deaf, 154(5), 417-420. https://doi.org/10.1353/aad.0.0115

Paul, P. V. y Moores, D. (2012). Deaf epistemologies. Multiple perspectives on the acquisition of on knowledge. Washington, DC: Gallaudet University Press.

Pérez Rodríguez, G. (1996). Metodología de la investigación educacional. La Habana: Pueblo y Educación. 
Piñar, P., Dussias, P. y Morford, J. P. (2011). Deaf readers as bilinguals: An examination of deaf readers print comprehension in light of current advances in bilingualism and second language processing. Language and Lingutics, 5(10), 691-704.

Robinson, O. y Henner, J. (2017). The personal is political in the deaf mute howls: Deaf epistemology seek disability justice. Disability ESociety, 32(9), 1416-1436. https://doi.org/10.1080/09687599.2017.1313723

Shulman, L. S. (1987). Knowledge and teaching: Foundations of the new reform. Harvard Educational Review, 57(1), 1-22. https://doi.org/10.17763/haer.57.1.j463w79r56455411

Vigotsky, L. S. (1978). Fundamentos de defectología. Madrid: Visor.

Young, A. y Temple, B. (2014). Approaches to social research. The case of deaf studies. Nueva York, NY: Oxford University Press. https://doi.org/10.1093/acprof:osobl/9780199929535.001.0001

Walsh, C. (2005). La interculturalidad en la educación. Lima: UNICEF.

\section{Breve CV de las autoras}

\section{Valeria Herrera Fernández}

Profesora Asociada en el Departamento de Educación de la Universidad de Chile. Doctora en Psicología por la Universidad Complutense de Madrid, España. Profesora en Educación Diferencial mención audición y lenguaje y Licenciada en Educación por la Universidad Metropolitana de Ciencias de la Educación, Chile. Sus investigaciones y más de 30 publicaciones abordan los procesos de enseñanza y aprendizaje del lenguaje escrito en estudiantes sordos desde un enfoque intercultural bilingüe. ORCID ID: https://orcid.org/1000-0001-8176-6195. Email: valherrera@u.uchile.cl

\section{Verónica de la Paz Calderón}

Directora ejecutiva del Instituto de la Sordera. Magister en Educación mención Multiculturalidad por la Universidad Academia de Humanismo Cristiano, Chile. Educadora Diferencial en Trastornos de Audición y Lenguaje por la Universidad de Chile. Ha dedicado su trabajo al reconocimiento de los derechos lingüísticos y culturales de los estudiantes sordos en distintas escuelas del país. Sus publicaciones se relacionan con Cultura sorda y educación intercultural bilingüe. ORCID ID: https://orcid.org/0000021165-0469. Email: vdelapaz@institutodelasordera.cl 